\title{
Correlação dos componentes vegetativos da cultura da pupunha e dos atributos químicos do solo
}

\author{
Rafael Montanari ${ }^{1}$, Elizeu de Souza Lima ${ }^{1}$, Lenon Henrique Lovera ${ }^{1}$, Samuel Ferrari ${ }^{2}$, \\ Hernandes Andrade Queiroz ${ }^{1}$
}

${ }^{1}$ Universidade Estadual Paulista "Júlio de Mesquita Filho"- FEIS-UNESP, Campus de Ilha Solteira, , Ilha Solteira, São Paulo, Brasil. E-mail: rafamontana@hotmail.com, elizeu.florestal@gmail.com, langoisa@gmail.com

${ }^{2}$ Universidade Estadual Paulista "Júlio de Mesquita Filho", Campus de Registro, Registro, São Paulo, Brasil. E-mail: ferrari@ registro.unesp.br

Recebido: 27/01/2016; Aceito: 01/06/2016.

\section{RESUMO}

\begin{abstract}
A pupunha (Bactris gasipaes) tem se mostrado uma excelente opção econômica para os produtores rurais, pois sua produção de palmito permite precocidade de corte, rusticidade, perfilhamento abundante, boa palatabilidade, ausência de oxidação do palmito, alta produtividade e facilidade nos tratos culturais. Objetivou-se selecionar, entre os atributos do solo pesquisado, aquele com a melhor correlação, linear e espacial, para explicar a variabilidade da planta da pupunha. O experimento foi conduzido na fazenda de pesquisa e extensão da Faculdade de Engenharia de Registro (SP), foi avaliada a variabilidade espacial dos componentes vegetativos da pupunha e dos atributos químicos de um Cambissolo Eutrófico. Uma malha contendo 54 pontos foi georreferenciada de forma a representar toda a área de amostragem de aproximadamente 1 ha. Os atributos químicos apresentaram variabilidade de muito alta a baixa e os atributos de planta apresentaram variabilidade de média a muito alta. Os valores médios de MO, $\mathrm{P}$, $\mathrm{Ca}$ e $\mathrm{Mg}$ encontrados estiveram próximos ao adequado para o desenvolvimento da pupunha, sendo explicado por um modelo exponencial e esférico altamente significativo em função do potássio e da CTC.
\end{abstract}

Palavras-chave: Bactris gasipaes, manejo do solo, geoestatística, Vale do Ribeira.

\section{Correlation of following components of culture peach palm and some chemical attributes of eutrochrept}

\begin{abstract}
The peach palm (Bactris gasipaes) has proven a great economical option for farmers because their palm production allows cutting precocity, rusticity, abundant tillering, tasty, no palm rust, high productivity and ease in cultivation. The objective was to select, between the soil properties, the one with the best correlation, linear and space to explain the variability of the peach palm plant. O experiment was conducted at the research farm, extent of Engineering College of Registro (SP) evaluated the spatial variability of the vegetative components of peach palm, and chemical properties of a Eutrophic Cambisol. A grid with 54 points was georeferenced to represent the entire sampling area of approximately 1.0 ha. Soil chemical properties presented variability from very high to low and plant properties had a mean variability too high. The average values of $\mathrm{MO}, \mathrm{P}, \mathrm{Ca}$ and $\mathrm{Mg}$ were found near suitable for the development of peach palm plant be explained by an exponential model is highly significant due to the spherical potassium and CTC.
\end{abstract}

Key words: Bactris gasipaes, Soil management, geoestathistical, Ribeira Valley. 


\section{Introdução}

A pupunha (Bactris gasipaes Kunth) é uma palmeira originária da Região Amazônica e, tem se mostrado uma excelente opção econômica para os produtores rurais, para a produção do palmito, devido ao seu potencial de mercado, além da promoção do reflorestamento sustentável e cobertura vegetal do solo (CHAIMSOHN, 2000).

As vantagens para o cultivo destinado à produção de palmito é a precocidade de corte, rusticidade, perfilhamento abundante, boa palatabilidade, ausência de oxidação do palmito, alta produtividade, facilidade nos tratos culturais e vantagens ecológicas, que podem fazer a cultura ser conduzida a pleno sol, em áreas agrícolas tradicionais, sem nenhum dano às matas nativas, fato este de grande apelo comercial, principalmente para a exploração do palmito, visando o mercado externo. A produção de frutos possui diversas finalidades no consumo direto como alimento, extração de óleo, farinha e ração animal (ARKCOLL; AGUIAR, 1984; CLEMENT, 1989).

A pupunheira é bastante exigente quanto às propriedades físicas e químicas do solo. Em relação às propriedades químicas, há grande exigência nas fases vegetativa e reprodutiva quando a planta extrai grande quantidade de nutrientes do solo. No entanto, a magnitude das respostas à aplicação de fertilizantes depende de uma série de fatores relacionados à absorção, transporte e utilização dos nutrientes disponíveis e aplicados ao solo, sendo assim necessário o uso de adubações para maximizar a produção (BOVI, 2000).

A agricultura de precisão iniciou com o intuito de captar a variabilidade espacial da fertilidade prévia do solo, utilizando para isso uma amostragem programada e criteriosa (COELHO, 2003). De posse de todas as informações obtidas a partir da amostragem, parte-se para o tratamento direcionado da fertilidade do solo, aplicando corretivos e fertilizantes a taxas variáveis, buscando sanar problemas que podem vir a depreciar a qualidade e a produtividade da lavoura por falta ou excesso de nutrientes (MENEGATTI; MOLIN, 2004).

$\mathrm{O}$ conhecimento da variabilidade espacial dos atributos de solo e de cultura apresenta-se como ferramenta vantajosa para analisar a variabilidade de rendimento verificada e aperfeiçoar o manejo em áreas agrícolas por meio de um gerenciamento agrícola que leve em consideração informações pontuais de solo e de culturas (MENDES et al., 2008; AMADO et al., 2009). Isso é possível através do estabelecimento de zonas específicas de manejo visando a conservação do solo e aumento da produtividade.

Com o intuito de estabelecer um indicador de qualidade química do solo que represente o melhor manejo para uma boa produtividade em Registro,
Estado de São Paulo, Brasil, neste trabalho objetivou-se: a) analisar a variabilidade destes atributos; b) definir as correlações lineares e espaciais entre os componentes vegetativos da cultura da pupunha e os atributos químicos do solo. O trabalho foi realizado com base nos dados de número de folhas (NF), diâmetro (Diam), altura (Alt), perfilhos (perf) e atributos da fertilidade do solo

\section{Material e Métodos}

O experimento foi conduzido na Fazenda de Ensino, Pesquisa e Extensão da Faculdade de Engenharia de Registro, no município de Registro (SP), sob as coordenadas geográficas de $24^{\circ} 32^{\prime} 08^{\prime \prime}$ de latitude $\mathrm{S}$ e $47^{\circ} 51^{\prime} 55^{\prime \prime}$ de longitude $\mathrm{W}$, com precipitação pluvial média anual de $1.500 \mathrm{~mm}$ e temperatura média de $25^{\circ}$ C. O clima é do tipo Af, segundo Köppen-Geiger, caracterizado como tropical sem estação seca.

$\mathrm{O}$ relevo e os tipos de solos da região constituem-se de sistema de planícies e terraços fluviais do Ribeira do Iguape, descrito como terreno plano no baixo ribeira, de sedimentos modernos, em solos aluviais argilosos do tipo Cambissolos Eutróficos em áreas de montante e hidromórficos eutróficos em solos de planície.

O preparo do solo foi realizado por meio de subsolagem e gradagem aradora foram realizados em janeiro de 2012. Ainda em janeiro, realizou-se a correção do $\mathrm{pH}$ do solo com $1,5 \mathrm{Mg} \mathrm{ha}^{-1}$ de calcário dolomítico e em seguida foi feita gradagem niveladora.

A adubação de plantio foi feita com $150 \mathrm{~g}$ por cova da fórmula 04-14-08 e a de manutenção foi feita de acordo com a análise de solo. Foi aplicada no primeiro ano a adubação de cobertura com $60 \mathrm{~kg} \mathrm{ha}^{-1} \mathrm{~N} ; 30 \mathrm{~kg}$ ha $^{-1} \mathrm{P}_{2} \mathrm{O}_{5}$ e $40 \mathrm{~kg} \mathrm{ha}^{-1} \mathrm{~K}_{2} \mathrm{O}+1250 \mathrm{~g} \mathrm{ha}^{-1}$ de FTE banana (Ca $7 \%$, S 5,7\%, B $1 \%, \mathrm{Cu} 1 \%$, Mn $1 \%$, Mo 0,1\% e Zn $18 \%$ ), sendo todas as aplicações ao redor da planta. No segundo ano foi aplicado $100 \mathrm{~kg} \mathrm{ha}^{-1} \mathrm{~N}, 50 \mathrm{~kg} \mathrm{ha}^{-1}$ $\mathrm{P}_{2} \mathrm{O}_{5}$ e $60 \mathrm{~kg} \mathrm{ha}^{-1} \mathrm{~K}_{2} \mathrm{O}+100 \mathrm{~g} \mathrm{ha}^{-1}$ de FTE banana e calcário $1000 \mathrm{~kg} \mathrm{ha}^{-1}$. O plantio das mudas foi realizado em 24 de janeiro de 2012, no Campus Experimental da UNESP na cidade de Registro, Estado de São Paulo; as coletas de planta e solo foram realizadas dia 25 de abril de 2014.

O espaçamento entre plantas utilizado para a instalação do experimento foi de $2,0 \times \mathrm{x} \quad 1,0 \mathrm{~m}$ (entrelinhas $\mathrm{x}$ planta). Foi instalada uma malha contendo 54 pontos de amostragem georreferenciada com o auxílio de um GPS Garmin, com intuito de representar toda a área de aproximadamente 1 ha, sendo a coleta realizada no entorno da planta.

Para a medição da altura (Alt) da planta utilizou-se uma régua graduada de $2 \mathrm{~m}$. O diâmetro (Diam) foi coletado a uma altura de $50 \mathrm{~cm}$ do solo com o auxílio de um paquímetro digital. A contagem do número de 
folhas (NF) e perfilhos (Perf) foi realizada manualmente.

As amostras foram coletadas nas profundidades 0,00-0,10 e $0,10-0,20 \mathrm{~m}$, e posteriormente encaminhadas ao laboratório para realizar as seguintes determinações: fósforo $(\mathrm{P})$, teor de matéria orgânica (MO), potencial hidrogeniônico $(\mathrm{pH})$, potássio $\left(\mathrm{K}^{+}\right)$, cálcio $\left(\mathrm{Ca}^{2+}\right)$, magnésio $\left(\mathrm{Mg}^{2+}\right)$, acidez potencial $(\mathrm{H}+\mathrm{Al})$, alumínio $\left(\mathrm{Al}^{3+}\right)$, soma de bases (SB), capacidade de troca catiônica (CTC), saturação por bases (V\%) e saturação por alumínio $(\mathrm{m} \%)$, conforme metodologia descrita por Raij et al. (1991).

Para cada atributo estudado, efetuou-se a análise descritiva clássica, com auxílio do software estatístico SAS Schlotzhaver e Littell (1997), em que foram calculados a média, mediana, valores mínimos e máximos, desvio-padrão, coeficiente de variação, curtose, assimetria e distribuição de frequência. Posteriormente, realizou-se a identificação dos outliers, efetuando a substituição dos seus valores pelo valor médio dos circunvizinhos contidos na malha.

Para testar a hipótese de normalidade ou de lognormalidade foi utilizada a estatística de Shapiro \& Wilk a 5\%. Também, foi montada a matriz de correlação, objetivando efetuar as correlações lineares simples para as combinações, duas a duas, entre todos os atributos estudados, assim como apresentar as análises de regressões para os pares de maior interesse. Desta forma, selecionaram-se aqueles de maior correlação linear e que, portanto, poderiam apresentar semivariograma cruzado e a consequente co-krigagem. Isoladamente para cada atributo, foi analisada sua dependência espacial, pelo cálculo do semivariograma simples. Contudo, para aqueles que apresentaram interdependência espacial, calcularam-se também seus semivariogramas cruzados, com base nos pressupostos de estacionaridade da hipótese intrínseca, pelo uso do pacote Gamma Design Software $\left(\mathrm{GS}^{+}, 2004\right)$.

Os ajustes dos semivariogramas simples e cruzados, em função de seus modelos, foram efetuados prioritariamente pela seleção inicial de: a) menor soma dos quadrados dos desvios (RSS); b) maior coeficiente de determinação $\left(r^{2}\right)$; c) maior avaliador da dependência espacial (ADE). A decisão final do modelo que representou o ajuste foi realizada pela validação cruzada, assim como para a definição do tamanho da vizinhança que proporcionou a melhor malha de krigagem e/ou co-krigagem, realizadas por meio da krigagem em blocos. Para cada atributo, foram relacionados o efeito pepita (Co), o alcance (Ao) e o patamar $(\mathrm{Co}+\mathrm{C})$. A análise do avaliador da dependência espacial (ADE) foi efetuada conforme a seguinte expressão (GS+, 2004):

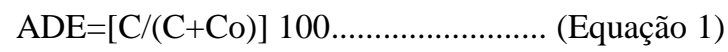

Em que:
ADE é o avaliador da dependência espacial; $\mathbf{C}$, a variância estrutural; e C + Co, o patamar.

A interpretação proposta para o ADE foi a seguinte: $\mathrm{ADE}(\leq 20 \%$ variável espacial com muito baixa dependência; $20 \%<\mathrm{ADE} \leq 40 \%$ variável espacial com baixa dependência; $40 \%<\mathrm{ADE} \leq 60 \%$ variável espacial com média dependência; $60 \%<\mathrm{ADE} \leq 80 \%$ variável espacial com alta dependência e de $80 \%$ < $\mathrm{ADE} \leq 100 \%$ variável espacial com muito alta dependência (DALCHIAVON; CARVALHO, 2012).

Por outro lado, sabe-se que a validação cruzada é uma ferramenta destinada a avaliar modelos alternativos de semivariogramas simples e cruzados, que respectivamente efetuarão a krigagem e a co-krigagem. $\mathrm{Na}$ sua análise, cada ponto contido dentro do domínio espacial foi removido individualmente, sendo seu valor estimado como se não existisse. Dessa forma, pôde-se construir um gráfico de valores estimados versus observados para todos os pontos. O coeficiente de correlação (r) entre tais valores refletiu a eficiência do ajuste, dado pela técnica da soma dos quadrados dos desvios, que representou a equação de regressão linear $\left(\mathrm{GS}^{+}, 2004\right)$. Assim, trabalhando-se na obtenção do número ideal de vizinhos, foram obtidos, por meio da interpolação, os mapas de krigagem e de co-krigagem, para análise da dependência e da interdependência espacial entre os atributos. Os componentes geoestatísticos determinados foram o semivariogramas simples, semivariograma cruzado, validação cruzada, krigagem e a co-krigagem.

\section{Resultados e Discussão}

$\mathrm{Na}$ Tabela 1, o número de folhas (NL) apresentou média variabilidade, com o coeficiente de variação de 19,7 \%. Já para diâmetro (Diam), altura (Alt) e perfilho (Perf) apresentaram grande variabilidade, com coeficientes de 33,8, 36,2 e 41,5\%, respectivamente. Segundo Pimentel-Gomes \& Garcia (2002), a variabilidade de um atributo pode ser classificada segundo a magnitude do seu coeficiente de variação (CV). Suas classes foram determinadas como baixa (CV $<10 \%)$, média $(10 \%<\mathrm{CV}<20 \%)$, alta $(20 \%<\mathrm{CV}<$ $30 \%)$ e muito alta (CV > 30\%).

Para os atributos K (51,2 \%; 41,9\%), Ca (48,9\%; $45,6 \%), \operatorname{Mg}(62,3 \% ; 61,4 \%), \mathrm{Al}(94,3 \% ; 51,2 \%), \mathrm{SB}$ $(52,9 \% ; 48,2 \%)$, v\% (42,6\%; 45,1\%) e m\% (97,3\%; $55,5 \%)$ em todas as profundidades e para $\mathrm{P}(36,0 \%)$ na segunda camada e $\mathrm{H}+\mathrm{Al}(35,7 \%)$ na primeira camada apresentaram muito alta variabilidade. Os atributos $\mathrm{P}$ 0,00-0,10 $\mathrm{m}$ e $\mathrm{H}+\mathrm{Al} 0,10-0,20 \mathrm{~m}$ apresentaram alta variabilidade. Já a CTC em ambas as profundidades, MO de 0,10-0,20 m; pH de 0,00-0,10 m apresentaram média variabilidade; os demais atributos apresentaram baixa variabilidade. 
Tabela 1. Análise descritiva dos componentes vegetativos da cultura da pupunheira e alguns atributos químicos de Cambissolo em Registro, Estado de São Paulo, 2014

\begin{tabular}{|c|c|c|c|c|c|c|c|c|c|c|}
\hline \multirow{2}{*}{ Atributos ${ }^{(1)}$} & \multirow{2}{*}{ Média } & \multirow{2}{*}{ Mediana } & \multicolumn{2}{|c|}{ Valor } & \multirow{2}{*}{$\begin{array}{l}\text { Desvio } \\
\text { Padrão }\end{array}$} & \multicolumn{3}{|c|}{ Coeficientes } & \multicolumn{2}{|c|}{$\begin{array}{c}\text { Teste de } \\
\text { probabilidade } \\
{ }^{(2)}\end{array}$} \\
\hline & & & Mínimo & Máximo & & $\begin{array}{c}\text { Variação } \\
(\%)\end{array}$ & Curtose & Assimetria & $\operatorname{Pr}<w$ & DF \\
\hline \multicolumn{11}{|c|}{ Atributos da planta } \\
\hline NF & 5,611 & 6,0 & 3,0 & 8,0 & 1,106 & 19,7 & 0,044 & $-0,118$ & 0,0081 & IN \\
\hline $\operatorname{Diam}(\mathrm{cm})$ & 6,342 & 6,5 & 2,1 & 13,0 & 2,632 & 41,5 & $-0,478$ & 0,315 & 0,2076 & NO \\
\hline Alt (m) & 1,169 & 1,140 & 0,5 & 2,180 & 0,395 & 33,8 & $-0,105$ & 0,497 & 0,2804 & NO \\
\hline Perf. & 6,703 & 7,0 & 2,0 & 12,0 & 2,431 & 36,2 & $-0,494$ & 0,220 & 0,1654 & NO \\
\hline \multicolumn{11}{|c|}{ Atributos do solo } \\
\hline $\mathrm{P} 1\left(\mathrm{mg} \mathrm{dm}^{-3}\right)$ & 6,296 & 6,000 & 4,0 & 12 & 1,839 & 29,2 & 0,578 & 0,829 & 0,0009 & IN \\
\hline $\mathrm{P} 2\left(\mathrm{mg} \mathrm{dm}^{-3}\right)$ & 4,000 & 4,000 & 2,0 & 8,0 & 1,441 & 36,0 & 1,188 & 1,061 & 0,0001 & IN \\
\hline $\operatorname{MO1}\left(\mathrm{g} \mathrm{dm}^{-3}\right)$ & 18,648 & 19,00 & 15 & 23 & 1,824 & 9,8 & 0,011 & 0,218 & 0,1181 & NO \\
\hline $\operatorname{MO} 2\left(\mathrm{~g} \mathrm{dm}^{-3}\right)$ & 15,796 & 16,00 & 10 & 22 & 2,513 & 15,9 & $-0,237$ & 0,136 & 0,4436 & NO \\
\hline $\mathrm{pH} 1$ & 4,824 & 4,600 & 4,0 & 6,1 & 0,573 & 11,9 & $-0,296$ & 0,899 & 0,0001 & IN \\
\hline $\mathrm{pH} 2$ & 4,354 & 4,300 & 4,0 & 5,0 & 0,201 & 4,6 & 1,222 & 0,875 & 0,0065 & IN \\
\hline $\mathrm{K} 1\left(\mathrm{mmol}_{\mathrm{c}} \mathrm{dm}^{-3}\right)$ & 1,772 & 1,650 & 0,6 & 3,8 & 0,907 & 51,2 & $-0,748$ & 0,581 & 0,0015 & IN \\
\hline $\mathrm{K} 2\left(\mathrm{mmol}_{\mathrm{c}} \mathrm{dm}^{-3}\right)$ & 0,991 & 1,000 & 0,4 & 1,8 & 0,415 & 41,9 & $-1,100$ & 0,275 & 0,0050 & IN \\
\hline $\mathrm{Ca} 1\left(\mathrm{mmol}_{\mathrm{c}} \mathrm{dm}^{-3}\right)$ & 19,056 & 16,000 & 5,0 & 42 & 9,316 & 48,9 & $-0,114$ & 0,870 & 0,0005 & IN \\
\hline $\mathrm{Ca} 2\left(\mathrm{mmol}_{\mathrm{c}} \mathrm{dm}^{-3}\right)$ & 11,333 & 10,500 & 3,0 & 23 & 5,169 & 45,6 & $-0,563$ & 0,579 & 0,0089 & IN \\
\hline $\operatorname{Mg} 1\left(\mathrm{mmol}_{\mathrm{c}} \mathrm{dm}^{-3}\right)$ & 16,093 & 12,500 & 3,0 & 45 & 10,021 & 62,3 & 0,672 & 1,096 & 0,0002 & IN \\
\hline $\operatorname{Mg} 2\left(\mathrm{mmol}_{\mathrm{c}} \mathrm{dm}^{-3}\right)$ & 9,537 & 8,000 & 2,0 & 26 & 5,853 & 61,4 & 1,013 & 1,184 & 0,0001 & IN \\
\hline $\mathrm{H}+\mathrm{All}\left(\mathrm{mmol}_{\mathrm{c}} \mathrm{dm}^{-3}\right)$ & 40,074 & 43,500 & 16 & 72 & 14,297 & 35,7 & $-0,434$ & 0,216 & 0,0256 & IN \\
\hline $\mathrm{H}+\mathrm{Al} 2\left(\mathrm{mmol}_{\mathrm{c}} \mathrm{dm}^{-3}\right)$ & 50,389 & 50,000 & 25 & 84 & 11,801 & 23,4 & 1,175 & 0,530 & 0,1094 & NO \\
\hline $\operatorname{Al} 1\left(\mathrm{mmol}_{\mathrm{c}} \mathrm{dm}^{-3}\right)$ & 5,315 & 5,000 & 0,0 & 20 & 5,012 & 94,3 & 0,411 & 0,923 & 0,0001 & IN \\
\hline $\mathrm{Al} 2\left(\mathrm{mmol}_{\mathrm{c}} \mathrm{dm}^{-3}\right)$ & 11,000 & 10,500 & 0,0 & 24 & 5,637 & 51,2 & $-0,640$ & 0,216 & 0,4373 & NO \\
\hline $\mathrm{SB} 1\left(\mathrm{mmol}_{\mathrm{c}} \mathrm{dm}^{-3}\right)$ & 37,013 & 29,600 & 8,8 & 88,9 & 19,596 & 52,9 & 0,212 & 0,978 & 0,0001 & IN \\
\hline $\mathrm{SB} 2\left(\mathrm{mmol}_{\mathrm{c}} \mathrm{dm}^{-3}\right)$ & 21,583 & 20,500 & 5,7 & 50 & 10,399 & 48,2 & 0,109 & 0,820 & 0,0001 & IN \\
\hline $\mathrm{CTC} 1\left(\mathrm{mmol}_{\mathrm{c}} \mathrm{dm}^{-3}\right)$ & 77,943 & 75,300 & 51,6 & 110,9 & 12,307 & 15,8 & 0,961 & 0,985 & 0,0007 & IN \\
\hline CTC2 $\left(\mathrm{mmol}_{\mathrm{c}} \mathrm{dm}^{-3}\right)$ & 72,655 & 72,150 & 53,1 & 104,7 & 10,907 & 15,0 & 1,116 & 0,834 & 0,0322 & IN \\
\hline $\mathrm{V} 1(\%)$ & 47,500 & 41,000 & 11 & 88 & 20,255 & 42,6 & $-0,867$ & 0,347 & 0,0456 & IN \\
\hline V2 (\%) & 30,296 & 28,000 & 6,0 & 60 & 13,650 & 45,1 & $-0,591$ & 0,547 & 0,0165 & IN \\
\hline m1 (\%) & 15,426 & 14,000 & 0,0 & 56 & 15,009 & 97,3 & 0,551 & 0,987 & 0,0001 & IN \\
\hline m2 (\%) & 35,852 & 34 & 0,0 & 78 & 19,906 & 55,5 & $-0,890$ & 0,122 & 0,3784 & NO \\
\hline
\end{tabular}

(1)NF, Diam, Alt Perf, são, respectivamente, o número de folhas, diâmetro, altura e Perfilhos; P, MO, pH, K, Ca, Mg, H+Al, Al, SB,CTC, V\% e m\%, 1 e 2, são, respectivamente, o fósforo, matéria orgânica, potencial hidrogeniônico, potássio, magnésio, acidez potencial, alumínio, soma de bases, capacidade de troca de capacidade, saturação por bases, saturação por alumínio, nas profundidades de 0-0,10 e 0,10-0,20 m; (2) DF: distribuição de frequência; NO e IN representam, respectivamente, normal e indeterminado.

Assim, para $\mathrm{MO}$ nas duas profundidades e $\mathrm{pH}, \mathrm{H}+\mathrm{Al}$ de 0,00 a $0,10 \mathrm{~m}$, foi diferente daqueles obtidos por Montanari et al. (2013a) que avaliando um Latossolo Vermelho aluminoférrico encontraram valores de alta variabilidade para $\mathrm{MO}$ nas duas profundidades entre 20,1 a 24,6 \%, baixa variabilidade para pH1 e média variabilidade para $\mathrm{H}+\mathrm{Al}$ de $0,00-0,10$ para os atributos estudados. Já para pH e H+Al na camada de 0,10-0,20 corroboram com valores encontrados pelo respectivo autor. Para os atributos $\mathrm{K}, \mathrm{Ca}$ e $\mathrm{Mg}$ em ambas as profundidades corroboram com aqueles encontrados por Dalchiavon et al. (2011) que trabalhando em um Latossolo Vermelho distroférrico, também encontraram muito alta variabilidade, variando entre 31,9 a $38,5 \%$.
Quando uma variável estatística qualquer possuir distribuição de frequência do tipo normal, a medida de tendência central mais adequada para representá-la deve ser a média, em contrapartida, será representada pela mediana, ou pela média geométrica, caso seja do tipo lognormal (DALCHIAVON et al., 2011).

Portanto, a medida de tendência central que representará os atributos Diam, Alt, Perf, MO1, MO2, $\mathrm{H}+\mathrm{Al} 2$ e m\% deverá ser a média, em razão de terem apresentado distribuição de frequência do tipo normal, com coeficientes de assimetria positiva, entre 0,122 e 0,530. Já os coeficientes de curtose positiva ficaram entre 0,011 e 1,175, e da negativa, com valores entre 0,105 e $-0,890$. 
Contudo, e independentemente desses coeficientes, foram significativos a $5 \%$ pelo teste de normalidade de Shapiro e Wilk, uma vez que essa probabilidade variou entre 0,1094 e 0,4373 (Tabela1).

Tendo por base os estudos geoestatísticos em que se analisaram as correlações entre os componentes vegetativos médios da pupunha e os atributos químicos do solo, em relação ao presente trabalho, o Diam e o Alt obtiveram médias de $6,342 \mathrm{~cm}$ e 1,169 m (Tabela 1).

Os teores médios de $\mathrm{P}$ (P1 de 6,296 $\mathrm{mg} \mathrm{dm}^{-3}, \mathrm{P} 2$ de 4,000 $\mathrm{mg} \mathrm{dm}^{-3}$ ), MO (MO1 de 18,648 $\mathrm{g} \mathrm{dm}^{-3}$ e MO2 de $\left.15,796 \mathrm{~g} \mathrm{dm}^{-3}\right), \mathrm{pH}(\mathrm{pH} 1 \mathrm{de} 4,824$ e pH2 de 4,354), K (K1 de 1,772 $\mathrm{mmol}_{\mathrm{c}} \mathrm{dm}^{-3}$ e K2 de 0,991 $\mathrm{mmol}_{\mathrm{c}} \mathrm{dm}^{-3}$ ), Ca (Ca1 de 19,056 $\mathrm{mmol}_{\mathrm{c}} \mathrm{dm}^{-3}$ e Ca2 de 11,333 $\mathrm{mmol}_{\mathrm{c}} \mathrm{dm}^{-}$ $\left.{ }^{3}\right)$ e $\mathrm{Mg}\left(\mathrm{Mg} 1\right.$ de $16,093 \mathrm{mmol}_{\mathrm{c}} \mathrm{dm}^{-3}$ e $\mathrm{Mg} 2$ de 9,537 $\mathrm{mmol}_{\mathrm{c}} \mathrm{dm}^{-3}$ ) sofreram uma diminuição em profundidade devido à adubação ter sido realizada em torno da planta em superficie. Canellas et al. (2003) estudando em um Cambissolo Háplico em cultivo de cana Queimada, encontraram teores semelhantes de $\mathrm{P}\left(6,3 \mathrm{mg} \mathrm{dm}^{-3}\right)$ e $\mathrm{K}$ $\left(2 \mathrm{mmol}_{\mathrm{c}} \mathrm{dm}^{-3}\right)$, porém, diferiram para $\mathrm{pH}, \mathrm{Ca}$ e $\mathrm{Mg}$ $\left(6,5 ; 48 \mathrm{mmol}_{\mathrm{c}} \mathrm{dm}^{-3} ; 29 \mathrm{mmol}_{\mathrm{c}} \mathrm{dm}^{-3}\right)$ respectivamente. Portugal et al. (2008), também estudando em um Cambissolo Háplico, encontraram valores semelhantes ao da presente pesquisa, de $\mathrm{P}$ e $\mathrm{pH}\left(7,91 \mathrm{mg} \mathrm{dm}^{-3} ; 4,60\right)$ respectivamente, e diferiram de $\mathrm{K}, \mathrm{Ca}$ e $\mathrm{Mg}$ (5,2 $\mathrm{mg} \mathrm{dm}^{-}$ ${ }^{3} ; 6,9 \mathrm{mmol}_{\mathrm{c}} \mathrm{dm}^{-3} ; 2,8 \mathrm{mmol}_{\mathrm{c}} \mathrm{dm}^{-3}$ ) respectivamente.

A correlação entre os componentes vegetativos da pupunha (NF, Diam, Alt e Perf) e os atributos químicos do solo (Tabela 2) apresentou significância exclusivamente para os pares: NF x K1 ( $\left.\mathrm{r}=-0,300^{*}\right)$, Diam x P1 $\left(\mathrm{r}=0,290^{*}\right)$, Diam x K1 $\left(\mathrm{r}=-0,360^{* *}\right)$,
Diam x Al2 $(r=-0,294 *)$, Diam x CTC2 $\left(r=-0,295^{*}\right)$, Alt x P1 $\left(\mathrm{r}=0,280^{*}\right)$, Alt x K1 $\left(\mathrm{r}=-0,381^{* *}\right)$, Alt x Al2 $(r=-0,272 *)$ e Alt x CTC2 $\left(r=-0,306^{*}\right)$. Todas essas correlações foram fracas, com exceção de Diam x K1, que para ser correlação média, valor precisa ser maior que 0,30 (HOPKINS, 2002). Na Figura 1a, b, c e d foram apresentadas as equações de regressão principalmente para os atributos Diam, Alt, P1 e K1, dada pelos seguintes modelos:

a) linear direta de regressão:

$$
\text { Diam }=3,726+0,416 \mathrm{P} 1
$$$$
\text { Alt }=0,790+0,060 \text { P1 }
$$

(Equação 2)

(Equação 4)

b) linear inversa de regressão:

$$
\begin{array}{ll}
\text { Diam }=8,197+1,047 \mathrm{~K} 1 & (\text { Equação 3) } \\
\text { Alt }=1,464-0,166 \mathrm{~K} 1 & (\text { Equação 5) }
\end{array}
$$

em que, Diam é o Diâmetro $(\mathrm{cm})$, Alt $(\mathrm{m})$ é a altura da pupunha e P1 mg dm e $\mathrm{K} 1 \mathrm{mmol}_{\mathrm{c}} \mathrm{dm}^{-3}$ correspondem à Fósforo e Potássio respectivamente estabelecidas nas camadas entre 0,00-0,10 m. Suas correlações, ainda que baixas, foram altamente significativas. Assim, quando ocorrer o máximo valor da P1 (12 $\left.\mathrm{mg} \mathrm{dm}^{-3}\right)$ implicará nos máximos estimados de Diam e Alt (8,718 cm e 1,513 m). Já para regressão linear inversa, quando ocorrer os máximos valores de $\mathrm{K} 1$ (3,8 $\left.\mathrm{mmol}_{\mathrm{c}} \mathrm{dm}^{-3}\right)$, implicará nos mínimos estimados de Diam e Alt (12,2 cm e 0,83 m) (Figura 1).

Analisando-se a Figura 2, pode-se inferir que a CTC2 apresentou-se como bom indicador da qualidade química do solo como um adequado reservatório de nutrientes, para um aumento do número de folhas.

\begin{tabular}{|c|c|c|c|c|c|c|c|c|c|}
\hline \multicolumn{10}{|c|}{ Coeficiente de correlação $(b)$} \\
\hline Atributos $^{(a)}$ & NF & Diam & Alt & Perf & Atributos $^{(a)}$ & $\mathrm{NF}$ & Diam & Alt & Perf \\
\hline Diam & $0,697 * *$ & - & - & - & - & - & - & - & - \\
\hline Alt & $0,667 * *$ & $0,927 * *$ & - & - & - & - & - & - & - \\
\hline Perf & 0,188 & 0,115 & 0,120 & - & - & - & - & - & - \\
\hline $\mathrm{P} 1$ & 0,243 & $0,290 *$ & $0,280^{*}$ & 0,058 & $\mathrm{H}+\mathrm{Al} 1$ & 0,101 & $-0,097$ & $-0,041$ & 0,039 \\
\hline $\mathrm{P} 2$ & $-0,095$ & $-0,032$ & $-0,032$ & 0,123 & $\mathrm{H}+\mathrm{Al} 2$ & 0,162 & $-0,261$ & $-0,257$ & 0,049 \\
\hline MO1 & 0,099 & 0,091 & 0,042 & $-0,203$ & Al1 & 0,035 & $-0,053$ & $-0,010$ & 0,093 \\
\hline MO2 & $-0,015$ & 0,016 & 0,023 & $-0,020$ & $\mathrm{~A} 12$ & 0,112 & $-0,294 *$ & $-0,272 *$ & 0,025 \\
\hline pH1 & 0,140 & 0,141 & 0,081 & $-0,062$ & SB1 & 0,022 & $-0,067$ & $-0,124$ & $-0,073$ \\
\hline $\mathrm{pH} 2$ & 0,096 & 0,200 & 0,165 & $-0,098$ & SB2 & 0,120 & 0,043 & 0,006 & $-0,094$ \\
\hline K1 & $-0,300^{*}$ & $0,360 * *$ & $0,381 * *$ & 0,103 & CTC1 & 0,037 & $-0,057$ & $-0,119$ & $-0,121$ \\
\hline $\mathrm{K} 2$ & $-0,238$ & $-0,243$ & $-0,245$ & 0,082 & СТC2 & 0,153 & $-0,295^{*}$ & $-0,306^{*}$ & $-0,081$ \\
\hline $\mathrm{Ca} 1$ & 0,022 & $-0,037$ & $-0,107$ & $-0,101$ & V1 & 0,126 & 0,093 & 0,010 & $-0,108$ \\
\hline $\mathrm{Ca} 2$ & 0,139 & 0,131 & 0,069 & $-0,115$ & $\mathrm{~V} 2$ & 0,083 & 0,104 & 0,077 & $-0,124$ \\
\hline $\mathrm{Mg} 1$ & 0,046 & 0,064 & $-0,111$ & $-0,056$ & $\mathrm{~m} 1$ & 0,025 & 0,005 & 0,090 & 0,121 \\
\hline $\mathrm{Mg} 2$ & 0,027 & $-0,069$ & $-0,065$ & $-0,108$ & $\mathrm{~m} 2$ & 0,038 & $-0,151$ & $-0,129$ & 0,134 \\
\hline
\end{tabular}

Tabela 2. Matriz de correlação linear simples entre de componentes vegetativos da cultura da pupunheira e alguns atributos químicos de Cambissolo em Registro, Estado de São Paulo, 2014

(1) NF, Diam, Alt, Perf, são, respectivamente, o número de folhas, diâmetro, altura e Perfilhos; $\mathrm{P}, \mathrm{MO}, \mathrm{pH}, \mathrm{K}, \mathrm{Ca}$ e $\mathrm{Mg}, \mathrm{H}+\mathrm{Al}, \mathrm{Al}$, $\mathrm{SB}, \mathrm{CTC}, \mathrm{V} \%$ e m\% 1 e 2, são, respectivamente, o fósforo, matéria orgânica, potencial hidrogeniônico, potássio, cálcio e magnésio, acidez potencial, alumínio, soma de bases, capacidade de troca catiônica, saturação por bases e saturação por alumínio em profundidades de $0-0,10$ e $0,10-0,20 \mathrm{~m} ;{ }^{(2)} * *$ e *: significativo a 1 e $5 \%$, respectivamente. 


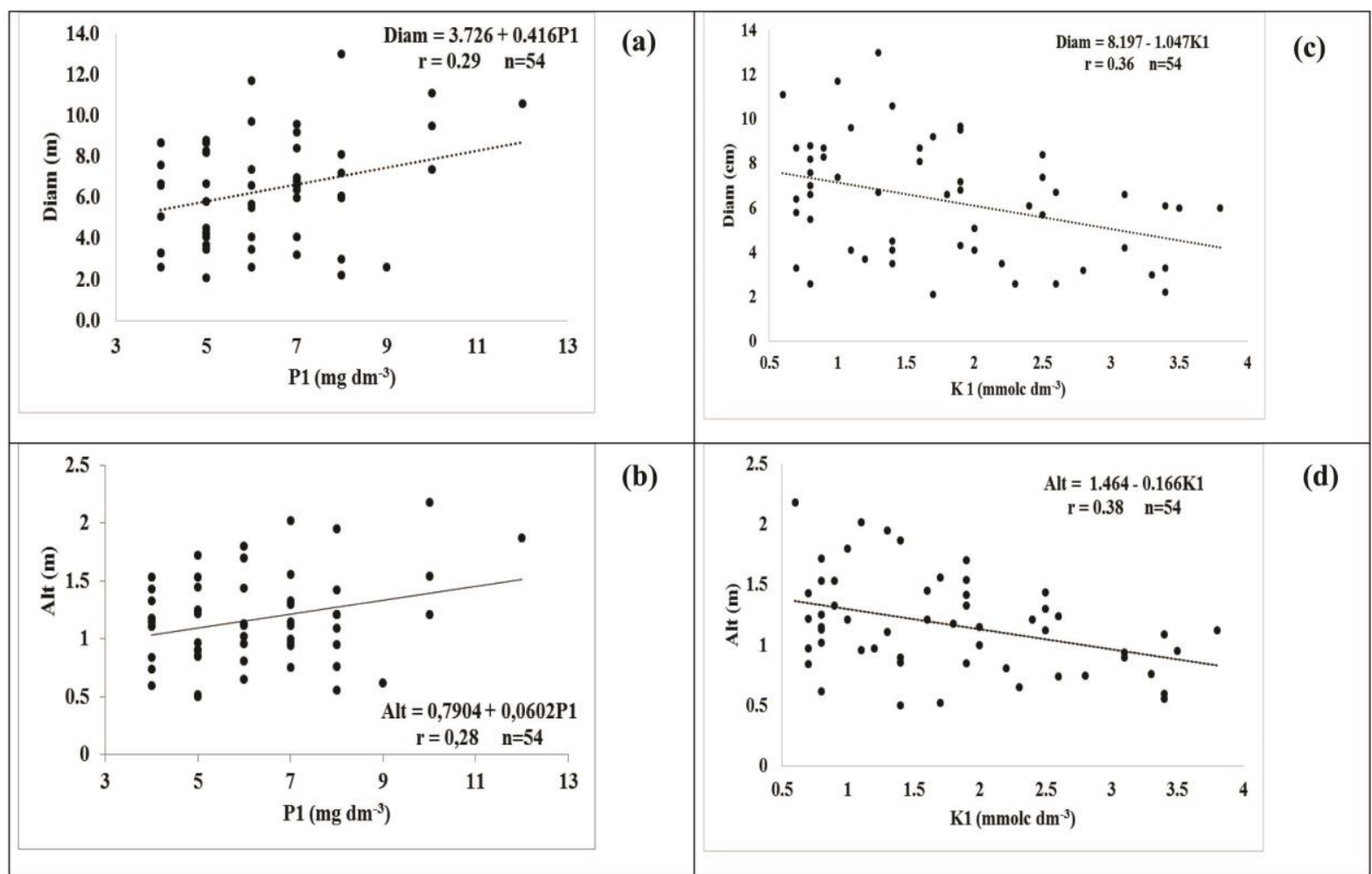

Figura 1. Equação de regressão entre Diam x P1(a); Alt x P1(b); Diam x K1(c) e Alt x K1(d) de um Cambissolo eutrófico no Vale do Ribeira em Registro (SP-Brasil), 2014.

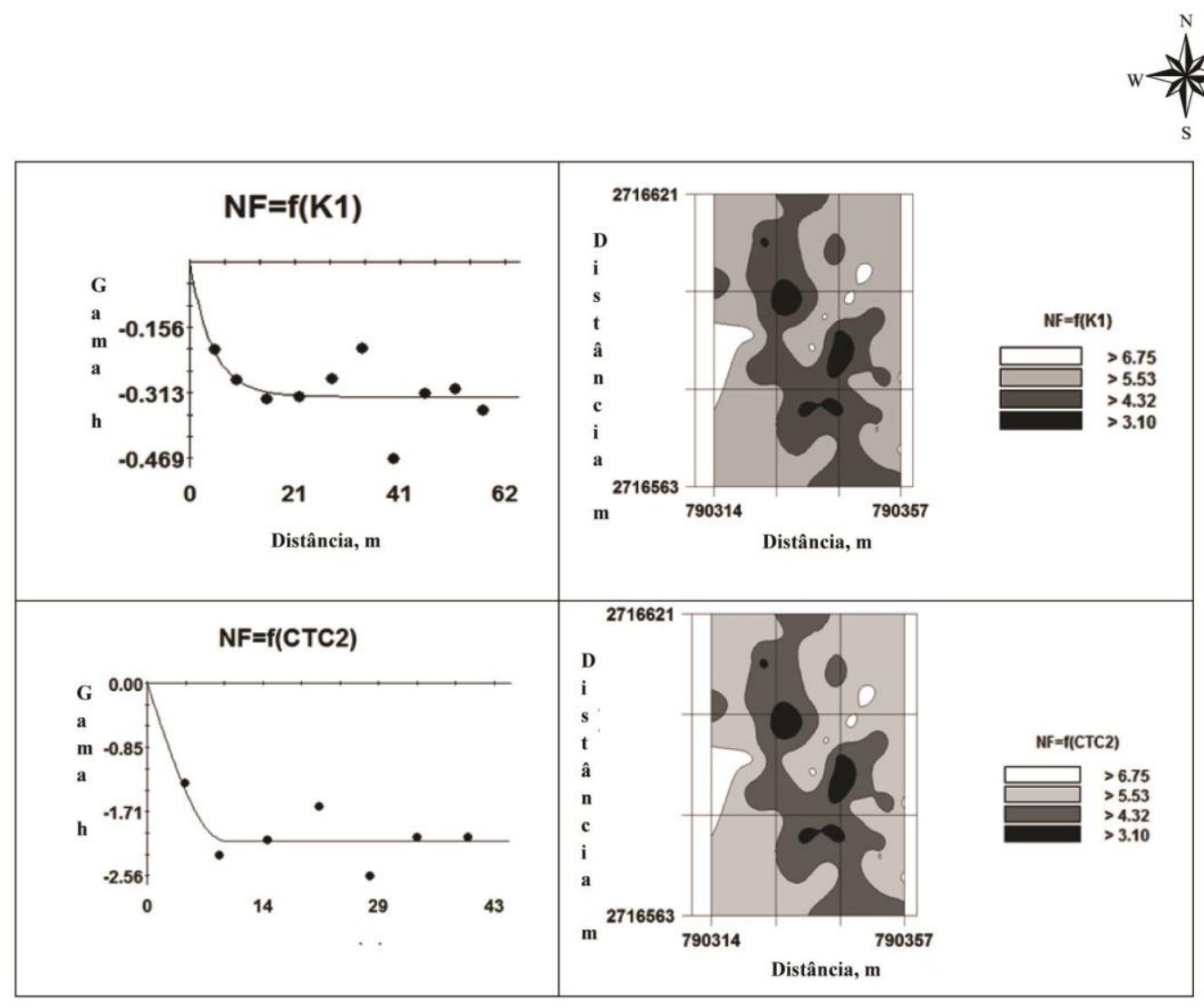

Figura 2. Semivariogramas cruzados e mapas de co-krigagem do número de folhas da pupunha em função do $\mathrm{K}$ de $0,00-0,10 \mathrm{~m}$ e CTC de 0,10-0,20 m de um Cambissolo Eutrófico no Vale do Ribeira em Registro (SP-Brasil) 
Quando realizado o semivariograma cruzado entre os atributos da planta, foram encontradas correlações espaciais negativas entre NF e CTC2 e NF e K1, em que se observou uma forte dependência espacial $(\mathrm{ADE}=$ 100,0 e 99,7 \%, respectivamente); o modelo ajustado foram o Esférico e Exponencial (Tabela 3 e Figura 2). Assim, como houve correlação linear significativa a $1 \%$ entre ambos, houve satisfatório ajuste semivariográfico.

Constatou-se, também, ajuste semivariográfico satisfatório entre a NF e a K1, pois houve correlação linear significativa a $1 \%$. Portanto, observando-se a Figura 2, pode-se inferir que o $\mathrm{K}$ mostrou-se também bom indicador da qualidade do solo, em relação à produção de número de folhas da pupunheira.

Tabela 3. Parâmetros de semivariogramas simples e cruzado dos componentes vegetativos da cultura da pupunheira e alguns atributos químicos de Cambissolo em Registro, Estado de São Paulo-SP

\begin{tabular}{|c|c|c|c|c|c|c|c|c|c|c|c|}
\hline \multirow{3}{*}{ Atributos ${ }^{\text {(a) }}$} & \multicolumn{11}{|c|}{ Parâmetros } \\
\hline & \multirow{2}{*}{$\begin{array}{l}\text { Modelo } \\
\text { (b) }\end{array}$} & \multirow{2}{*}{$\begin{array}{c}\text { Efeito } \\
\text { Pepita } \\
\left(\mathrm{C}_{0}\right) \\
\end{array}$} & \multirow{2}{*}{$\begin{array}{l}\text { Patamar } \\
\left(\mathrm{C}_{0}+\mathrm{C}\right)\end{array}$} & \multirow{2}{*}{$\begin{array}{l}\text { Alcance } \\
\left(\mathrm{A}_{0}\right)(\mathrm{m})\end{array}$} & \multirow[b]{2}{*}{$r^{2}$} & \multirow[b]{2}{*}{$\mathrm{SQR}^{(\mathrm{c})}$} & \multicolumn{2}{|c|}{$\mathrm{ADE}^{(\mathrm{d})}$} & \multicolumn{3}{|c|}{ Validação-cruzada } \\
\hline & & & & & & & $\%$ & Classe & a & $\mathrm{b}$ & $\mathrm{r}$ \\
\hline & \multicolumn{11}{|c|}{$\gamma(h)$ Atributos simples da planta } \\
\hline NF & $\exp (51)$ & $2,280 \times 10^{-1}$ & 1,367 & 13,30 & 0,972 & $3,430 \times 10^{-3}$ & 83,3 & MA & 2,150 & 0,620 & 0,330 \\
\hline Diam & $\operatorname{esf}(51)$ & $2,200 \times 10^{-1}$ & 7,126 & 8,43 & 0,894 & $2,960 \times 10^{1}$ & 96,9 & MA & 5,320 & 0,161 & 0,090 \\
\hline Alt & epp. & $1,532 \times 10^{-1}$ & $1,532 \times 10^{1}$ & - & - & - & - & - & - & - & - \\
\hline \multirow[t]{2}{*}{ Perf } & epp. & 6,56 & 6,56 & - & - & - & - & - & - & - & - \\
\hline & \multicolumn{11}{|c|}{$\gamma(h)$ Atributos simples de químico do solo } \\
\hline P1 & epp. & 3,41 & 3,41 & - & - & - & - & - & - & - & - \\
\hline $\mathrm{P} 2$ & epp. & 2,061 & 2,061 & - & - & - & - & - & - & - & - \\
\hline MO1 & exp. $(51)$ & $2,440 \times 10^{-1}$ & 2,964 & 9,09 & 0,530 & 0,213 & 91,8 & MA & $1,031 \times 10^{1}$ & 0,445 & 0,228 \\
\hline MO2 & epp. & 6,287 & 6,287 & - & - & - & - & - & - & - & - \\
\hline pH1 & exp. (51) & $3,660 \times 10^{-2}$ & $3,172 \times 10^{1}$ & 11,40 & 0,760 & $1,469 \times 10^{-3}$ & 88,5 & MA & $9,000 \times 10^{-1}$ & 0,818 & 0,413 \\
\hline $\mathrm{pH} 2$ & exp. (51) & $9,000 \times 10^{-3}$ & $4,620 \times 10^{-2}$ & 14,91 & 0,822 & $3,143 \times 10^{-5}$ & 80,5 & MA & 2,470 & 0,430 & 0,189 \\
\hline $\mathrm{K} 1$ & exp. (51) & $6,700 \times 10^{-2}$ & $7,600 \times 10^{-1}$ & 12,75 & 0,938 & $2,546 \times 10^{-3}$ & 91,2 & MA & 1,400 & 0,200 & 0,105 \\
\hline $\mathrm{K} 2$ & esf. (51) & $6,520 \times 10^{-2}$ & $1,934 \times 10^{-1}$ & 25,71 & 0,829 & $1,516 \times 10^{-3}$ & 66,3 & $\mathrm{AL}$ & $4,000 \times 10^{-2}$ & 0,956 & 0,628 \\
\hline $\mathrm{Ca} 1$ & esf. (51) & $8,700 \times 10^{5}$ & $8,110 \times 10^{4}$ & 12,45 & 0,279 & $1,260 \times 10^{-4}$ & 89,3 & MA & 5,680 & 0,697 & 0,351 \\
\hline $\mathrm{Ca} 2$ & exp. (51) & $2,990 \times 10^{4}$ & $2,551 \times 10^{3}$ & 14,40 & 0,219 & $1,200 \times 10^{4}$ & 88,3 & MA & 4,240 & 0,609 & 0,313 \\
\hline Mg1 & esf. (51) & $4,090 \times 10^{1}$ & $8,180 \times 10^{1}$ & 23,00 & 0,483 & $7,380 \times 10^{2}$ & 50,0 & ME & $-0,430$ & 1,035 & 0,457 \\
\hline $\mathrm{Mg} 2$ & esf. (51) & $1,539 \times 10^{1}$ & $3,079 \times 10^{1}$ & 28,20 & 0,386 & $2,270 \times 10^{2}$ & 30,0 & BA & 2,510 & 0,731 & 0,095 \\
\hline $\mathrm{H}+\mathrm{A} 11$ & epp. & $2,000 \times 10^{2}$ & $2,000 \times 10^{2}$ & - & - & - & - & - & - & - & - \\
\hline $\mathrm{H}+\mathrm{A} 12$ & epp. & $1,283 \times 10^{2}$ & $1,283 \times 10^{2}$ & - & - & - & - & - & - & - & - \\
\hline Al1 & epp. & $2,506 \times 10^{1}$ & $2,506 \times 10^{1}$ & - & - & - & - & - & - & - & - \\
\hline $\mathrm{Al} 2$ & epp. & $3,016 \times 10^{1}$ & $3,016 \times 10^{1}$ & - & - & - & - & - & - & - & - \\
\hline SB1 & exp. (51) & $5,200 \times 10^{1}$ & $3,96 \times 10^{2}$ & 13,20 & 0,267 & $2,759 \times 10^{1}$ & 86,9 & MA & $1,388 \times 10^{1}$ & 0,625 & 0,330 \\
\hline SB2 & exp. (51) & $1,000 \times 10^{1}$ & $1,071 \times 10^{2}$ & 13,80 & 0,230 & $3,399 \times 10^{3}$ & 90,7 & MA & $1,043 \times 10^{1}$ & 0,516 & 0,179 \\
\hline CTC1 & epp. & $1,335 \times 10^{2}$ & $1,335 \times 10^{2}$ & - & - & - & - & - & - & - & - \\
\hline CTC2 & esf. (48) & $4,940 \times 10^{6}$ & $1,029 \times 10^{7}$ & 21,60 & 0,653 & $7,440 \times 10^{2}$ & 52,0 & ME & $2,450 \times 10^{1}$ & 0,660 & 0,319 \\
\hline V1 & exp. (51) & $6,900 \times 10^{1}$ & $4,260 \times 10^{2}$ & 16,70 & 0,639 & $1,080 \times 10^{4}$ & 83,8 & MA & $1,175 \times 10^{1}$ & 0,758 & 0,367 \\
\hline $\mathrm{V} 2$ & exp. (49) & $5,180 \times 10^{1}$ & $1,670 \times 10^{2}$ & 14,70 & 0,597 & $1,016 \times 10^{3}$ & 69,0 & $\mathrm{AL}$ & $1,718 \times 10^{1}$ & 0,423 & 0,170 \\
\hline $\mathrm{m} 1$ & epp. & $2,289 \times 10^{2}$ & $2,289 \times 10^{2}$ & - & - & - & - & - & - & - & - \\
\hline \multirow[t]{2}{*}{$\mathrm{m} 2$} & exp. (44) & $7,900 \times 10^{1}$ & $4,040 \times 10^{2}$ & 14,10 & 0,761 & $3,113 \times 103$ & 80,5 & MA & $1,864 \times 10^{1}$ & 0,486 & 0,207 \\
\hline & \multicolumn{11}{|c|}{$y(h)$ planta $x$ solo } \\
\hline $\mathrm{NF}=\mathrm{f}(\mathrm{K} 1)$ & exp. (51) & $1,000 \times 10^{-3}$ & $3,220 \times 10^{-1}$ & 13,50 & 0,237 & $3,890 \times 10^{-2}$ & 99,7 & MA & 3,510 & 0,372 & 0,279 \\
\hline $\mathrm{NF}=\mathrm{f}(\mathrm{CTC} 2)$ & esf. (51) & $1,000 \times 10^{-3}$ & $-2,101$ & 9,73 & 0,522 & $4,840 \times 10^{-1}$ & 100,0 & MA & 3,510 & 0,372 & 0,279 \\
\hline
\end{tabular}

(1) NF, Diam, Alt, Perf, são, respectivamente, o número de folhas, diâmetro, altura e Perfilhos; $\mathrm{P}, \mathrm{MO}, \mathrm{pH}, \mathrm{K}, \mathrm{Ca}, \mathrm{Mg}, \mathrm{H}+\mathrm{Al}, \mathrm{Al}$, $\mathrm{SB}, \mathrm{CTC}, \mathrm{V} \%$ e m\%, 1 e 2, são, respectivamente, o fósforo, matéria orgânica, potencial hidrogeniônico, potássio, magnésio, acidez potencial, alumínio, soma de bases, capacidade de troca catiônica, saturação por bases, saturação por alumínio, nas profundidades de 0-0,10 e 0,10-0,20 m; parênteses após modelo: número de pares no primeiro lag; (2) esf: esférico, exp: exponencial, Gaus: gaussiano, (3) RSS: soma dos quadrados dos resíduos (4), ADE: avaliador da dependência espacial, e BA: baixa dependência, ME significa dependência, AL: alta dependência e MA : muito alta dependência. 
A análise geoestatística (Tabela 3) atestou que os atributos que apresentaram dependência espacial, analisada pela grandeza do coeficiente de determinação espacial $\left(\mathrm{r}^{2}\right)$, foi a seguinte: 1) NF $(0,972)$; 2) K1 $(0,938)$; 3) Diam (0,894); 4) K2 (0,829); 5) $\mathrm{pH} 2$ $(0,822)$; 6) $\mathrm{m} \% 2(0,761)$; 7) $\mathrm{pH} 1(0,860)$; 8) CTC2 $(0,653)$; 9) V\%1 (0,639); 10) V\%2 (0,597); 11) MO1 $(0,530)$; 12) $\operatorname{Mg} 1(0,483)$; 13) $\operatorname{Mg} 2(0,386)$; 14) Ca1 $(0,279)$; 15) SB1 (0,267); 16) SB2 $(0,230)$ e 17) Ca2 $(0,219)$.

Assim, em relação ao primeiro (NF), que teve elevado coeficiente de determinação espacial, observouse o seguinte: o seu valor do r2 $(0,979)$ indicou ser o atributo de melhor ajuste semivariográfico. Para o alcance do $\mathrm{pH} 1$ do presente trabalho $(11,4 \mathrm{~m})$ foi menor do que aquele encontrado por Montanari et al. (2013b), que foi de $16,5 \mathrm{~m}$. Já para o ADE, a forte dependência verificada $(\mathrm{ADE}=88,5 \%)$ encontrado também concordaram com o referido autor.

Na Tabela 3, observa-se a relação decrescente dos alcances da seguinte maneira: 1) $\operatorname{Mg} 2(28,2 \mathrm{~m})$; 2) K2 (25,71 m); 3) Mg1 (23,0 m); 4) CTC2 (21,6 m); 5) v\%1 (16,7 m); 6) pH2 (14,91 m); 7) v\%2 (14,70 m); 8) Ca2 (14,40 m); 9) m\%2 (14,10 m); 10) SB2 (13,8 m); 11) NF (13,3 m); 12) SB1 (13,2 m); 13) K1 (12,75 m); 14) Ca1 (12,45 m); 15) pH1 (11,40 m); 16) MO1 (9,09 m) e Diam $(8,43 \mathrm{~m})$. Portanto, nas condições da presente pesquisa e visando auxiliar pesquisas futuras, nas quais os mesmos atributos estejam envolvidos, os valores dos alcances a serem utilizados nos pacotes geoestatísticos, que alimentarão os pacotes computacionais empregados na agricultura de precisão, não deverão ser menores do que $8,43 \mathrm{~m}$.

Ainda quanto aos atributos Alt, Perf, P1, P2, MO2, $\mathrm{H}+\mathrm{Al} 1, \mathrm{H}+\mathrm{Al} 2, \mathrm{Al1}, \mathrm{Al} 2, \mathrm{CTC} 1 \mathrm{em} 1 \%$ apresentaram efeito pepita puro, sem dependência espacial

\section{Conclusões}

Os teores médios de matéria orgânica, fósforo cálcio e magnésio, nas respectivas profundidades foram considerados ideais para o desenvolvimento da pupunha.

Do ponto de vista linear, os componentes vegetativos da pupunha foram explicados por modelos exponenciais e esféricos altamente significativos, em função da presença do potássio e da CTC.

Os componentes vegetativos da pupunha podem ser estimados por meio da co-krigagem direta com o potássio. Assim, valores inferiores a $0,991 \mathrm{mmol}_{\mathrm{c}} \mathrm{dm}^{-3}$ desse atributo do solo indicaram sítios com redução na quantidade do número de folhas.

\section{Referências Bibliográficas}

AMADO, T. J. C.; PES, L. Z.; LEMAINSKI, C. L.; SCHENATO, R. B. Atributos químicos e físicos de Latossolos e sua relação com os rendimentos de milho e feijão irrigados. Revista Brasileira de Ciência do Solo, Viçosa-MG, v. 33, n. 4, p. 831-843, 2009.

ARKCOLL, D. B.; AGUIAR, J. P. L. Peach palm (Bactris gasipaes H.B.K.) a new source of vegetable oil from the wet tropics. Journal of the Science of Food and Agriculture, London-England, v. 35, n. 14, p. 520-526, 1984.

BOVI, M.L.A. O agronegócio palmito de pupunha. O Agronômico, Campinas-SP, v. 52, n. 1, p. 10-12, 2000.

CANELLAS, L. P.; VELLOSO, A.C.X.; MARCIANO, C. R; RAMALHO, J. F. G. P.; RUMJANEK, V. M.; REZENDE, C. E.; SANTOS, G. A. Propriedades químicas de um Cambissolo cultivado com cana-de-açúcar, com preservação do palhiço e adição de vinhaça por longo tempo. Revista Brasileira de Ciência do Solo, Viçosa-MG, v. 27, n. 5, p. 935-944, 2003.

CHAIMSOHN, F.P. Cultivo de pupunha e produção de palmito. Viçosa-MG: Aprenda Fácil, 2000. 121 p.

CLEMENT, C.R. The potencial use of the pejibaye palm in agroforestry systems. Agroforestry Systems Journal, Dordrecht-Netherlands, v. 7, n. 3, p. 201-212 1989.

COELHO, A.M. Agricultura de precisão: manejo da variabilidade espacial e temporal dos solos e das culturas. In: NOVAIS, R. F.; ALVAREZ, V. V. H.; SCHAEFER, C. E. G. R., (Eds.) Tópicos em Ciência do Solo. Viçosa-MG: Sociedade Brasileira de Ciência do Solo, 2003. p. 249-290.

DALCHIAVON, F. C.; CARVALHO, M. P. Correlação linear e espacial dos componentes de produção e produtividade da soja. Semina: Ciência Agrárias, Londrina-PR, v. 33, n. 2, p. 541-552, 2012.

DALCHIAVON, C. F.; CARVALHO, M. P.; FREDDI, O. S.; ANDREOTTI, M.; MONTANARI, R. Variabilidade espacial da produtividade do feijoeiro correlacionada com atributos químicos de um Latossolo Vermelho distroférrico sob sistema de semeadura direta. Bragantia, Campinas-SP, v. 70, n. 4, p. 908-916, 2011.

$\mathrm{GS}^{+}$. Geostatistics for environmental sciences. 7.ed. Michigan: Plainwell, Gamma Desing Software, 2004. 159p.

HOPKINS, W. G. A scale of magnitudes for effect statistics. A new view of statistics, 2002

MENDES, A. M. S.; FONTES, R. L. F.; OLIVEIRA, M. Variabilidade espacial da textura de dois solos do Deserto Salino, no Estado do Rio Grande do Norte. Revista Ciência Agronômica, Fortaleza-CE v. 39, n. 1, p. 19-27, 2008.

MENEGATTI, L. A. A.; MOLIN, J. P. Remoção de erros em mapas de produtividade via filtragem de dados brutos. Revista Brasileira de Engenharia Agrícola e Ambiental, Campina Grande-PB, v. 8, n. 1, p. 126-134, 2004.

MONTANARI, R.; CARVALHO, M. P.; TEIXEIRA FILHO, M. C. M.; DALCHIAVON, F. C. Produção de matéria seca da 
braquiária de acordo com os atributos químicos de um Latossolo em Selvíria, Mato Grosso do Sul. Revista Ceres, Viçosa-MG, v. 60, n. 4, p. 519-527, 2013a.

MONTANARI, R.; RODRIGUES, G. G. CARVALHO, M. P.; DALCHIAVON, F. C.; MACHADO, F. C.; GONZALEZ, A. P. Atributos químicos de um Latossolo espacialmente relacionados com a produtividade e componentes de produção do feijão em Selvíria (MS). Revista de Ciências Agroveterinárias, Lages-SC, v. 12, n. 3, p. 271-281, 2013 b.

PIMENTEL-GOMES, F. P.; GARCIA, C. H. Estatística aplicada a experimentos agronômicos e florestais. Piracicaba-SP: FEALQ, 2002. 309p.
PORTUGAL, A. F.; COSTA, O. D. V.; COSTA, L. M.; SANTOS, B. C. M. Atributos químicos e físicos de um cambissolo háplico Tb distrófico sob diferentes usos na Zona da Mata Mineira. Revista Brasileira de Ciência do Solo, Viçosa-MG, v. 32, n. 1, p. 249-258, 2008.

RAIJ, B. V; ANDRADE, J. C.; CANTARELLA, H.; QUAGIO, J. A. Análise química para avaliação da fertilidade de solos tropicais. Campinas-SP: Instituto Agronômico de Campinas, 1991. 284 p.

SCHLOTZHAVER, S. D.; LITTELL, R. C. SAS system for elementary statistical analysis. 2.ed. Cary: SAS, 1997. 441p. 